\title{
Research on Preventing Consecutive Collision Algorithm for Real Time Location Transmit-Only Tags
}

\author{
Tian Chuangeng ${ }^{1,2}, \mathrm{Xu} \mathrm{Zhao}^{1,{ }^{*}}$ and Tang Xiang ${ }^{2}$ \\ ${ }^{I}$ School of Information and Electrical Engineering, China University of Mining and Technology, Xuzhou, Jiangsu \\ 221008, China; \\ ${ }^{2}$ Department of Information and Electrical engineering, Xuzhou Institute of Technology, Xuzhou, Jiangsu 221018, China
}

\begin{abstract}
In the real-time locating system based on RFID technology used in underground coal mines, the active transmit-only tags are commonly used. When the identity number signals transmitted by several tags are overlapped in terms of the time, there will be collision, which may result in omitted reading of the card reader. Generally, the periodical transmission of tags will lead to consecutive collision, which will increase the omission rate. As such, this paper designed an Identity number Based Blink Interval Generating Algorithm (IBBIGA) that can be used to generate several blink intervals for each tag without a random number. This algorithm effectively prevents consecutive collision and guarantees an equal average blink interval of each tag. Through simulation analysis, compared with the blink interval generation method used in ALOHA protocol and INCTITS371.1 protocol, IBBIGA has a lower omission rate.
\end{abstract}

Keywords: RFID, transmit-only tags, omission rate.

\section{INTRODUCTION}

Real-time location system plays a very important role in the production management and response to emergencies in coal mines, and its main functions include real-time acquisition and display of specific locations of moving objects in the underground coal mine. The RFID technology is used to realize this $[1,2]$ by fixing the tags on the moving objects and determining the location of the objects according to the identity number sent by the tags and collected by the card readers distributed in the underground coal mine. In most cases, the active tags are used in the system to increase the identification distance [3,4], which means the tags will broadcast identity number actively and periodically. To guarantee a longer service time and lower cost of the tags, only transmit-only tags are used $[5,6]$.

When several objects are moving at the same time, the tags carried by those objects will blink out wireless signals that collide with each other, and this may make the card reader unable to receive the identity number transmitted by the tags, and may further result in omitted reading of the identity number and failure of the locating system. When several tags are at the same location, the mutual interference is inevitable. By checking the occupation of channel before sending the signal, the collision between tags could be mitigated [7]. The channel occupation can not be determined with a transmit-only tags, therefore, the tag blink frequency or card reading cycle should be increased to reduce the omission rate [5]. When the blink interval of the tags are the same, there will be consecutive collision of transmitted signals, which may result in long-term omission of identity number. As the transmit-only tags can not receive signals, the synchronous method can not be used to avoid collision [8]. Under such a case, the probability of consecutive collision of signals could only be lowered by changing the blink interval of tags. INCITS371.1 protocol specifies that the blink interval could be changed by extending or shortening the blink cycle by a random value upon transmission. The literatures [3,9] all use this method to generate a tag blink interval. The pure ALOHA protocol allows the tag to be connected at any time randomly [10]. In literature [11], the probability of collision caused by random blink interval is discussed. Because many tag hardware structures are not able to generate a random number, the method using a random blink interval is hard to be implemented during real practice. This paper analysed the conditions for consecutive collision, and proposed an algorithm for calculating blink intervals to avoid consecutive collision, which is the Identity number Based Blink Interval Generating Algorithm IBBIGA. IBBIGA has no random number, and it fully utilizes the uniqueness of the identity number and assigns a set of blink intervals to each tag. To ensure equal energy consumption of the tags in the same batch, the algorithm has to ensure that the average blink intervals of all tags are the same. The simulation result showed that the algorithm has a lower omission rate than the random generation of blink intervals. 


\section{CONSECUTIVE COLLISION MODEL OF TAG SIGNAL}

The card readers arranged in the underground coal mines are connected to the aboveground monitoring center through wire link. When the tag goes past the identification area of a certain card reader, the card reader can receive the wireless signal carrying an identity number sent out by the tag, indicating that the tag reaches the identification area of the card reader. If the card reader fails to receive the identity number within a card reading cycle after the identity number was received, then the tag will be regarded as having left the identification area. The card reader will sent the information on entry and exit of the tag to the aboveground monitoring center to track the location of the moving tags. If a tag stays in the same identification area for more than one card reading cycle while the card reader fails to receive the signal transmitted by the tag, this will be regarded as an omission. The reason for omission is that the card reader fails to receive the signal sent by the tag for several times within the card reading cycle. If a lot of tags are in the same place, there will be signal interference. In such a case, there will easily be omission.

The tag and card reader in the system work in the same wireless signal channel, and the card reading cycle of the card reader is $T_{r}$. The time during which the signal channel is occupied by the identity number sent by the tag is $t_{s}$. Any two tags (tags A and B) are located in the card reading area of a certain card reader for an extended period of time, with a transmission cycle of respectively $T_{t a}$ and $T_{t b}$, as shown in Fig. (1). There is a difference $\Delta t$ between the time of the first blink by two tags and $\Delta t \leq \max \left(T_{t a}, T_{t b}\right)$.

When collision is caused by overlap of the transmission times of the two tags, there will be integers $m$ and $n$, and then the inequation

$$
\left|m T_{t b}-n T_{t a}-\Delta t\right|<t_{s}
$$

will be established. In case of consecutive collision between two tags, the collision conditions will be met for $k$ cycles after the first collision, i.e. $\left|m T_{t b}-n T_{t a}+k\left(T_{t b}-T_{t a}\right)-\Delta t\right|<t_{s}$

According to the above two formulas, the conditions for the number of times of consecutive collision to reach $k+1$ should be:

$\left|T_{t b}-T_{t a}\right|<\frac{2 t_{s}}{k} \quad k \geq 1$

When, $\frac{T_{r}}{T_{t a}} \leq k+1$ or $\frac{T_{r}}{T_{t b}} \leq k+1$, there will be omission of identification card reading.

As known from Formula (3), when the absolute value of the difference between the transmission cycles of any two tags is less than 2 times of the transmission time, there will be consecutive collision. If it can be guaranteed that the absolute value of the difference between any two connection intervals of two tags is larger or equal to $2 t_{s}$, then it can be assured that there will not be any collision again during the next blink by the tags that collided before.

\section{IDENTITY NUMBER BASED BLINK INTERVAL GENERATING ALGORITHM}

\subsection{Algorithm Design}

When calculating the interval of each blink of the tag, the average interval should be determined at the first. The average interval is mainly limited by the two factors: running hour of the tag and the time it takes the tag to go through the identification area. In the some standards of the real-time locating system in the underground coal mine, there are specific indicators of the two parameters.

Assume the average interval of signal transmission by the tag is $\overline{T_{B I}}$, the energy consumption for each transmission is $E_{t}$ and the electric quantity of the battery carried by the active tag is $E$ and the minimum required working time of the tag is $T$. Then:

$$
\frac{\overline{T_{B I}} \times E}{E_{t}}>T \text {, i.e., } T_{B I}>\frac{T \times E_{t}}{E}
$$

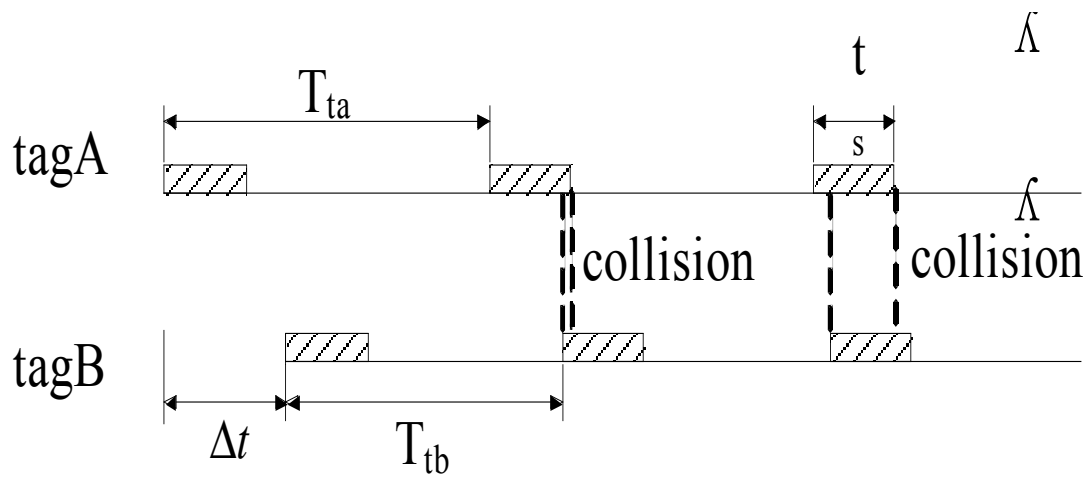

Fig. (1). Consecutive collision of periodically access. 
When a tag goes past the card reading range of a card reader, assume the maximum passing speed is $v$, and the radius of the identification area of the card reader is $d$, then the time it takes the tag to go past the identification area is $\frac{2 d}{v}$. The tag should be able to transmit signal at least for $n_{t}$ times during this period of time to ensure the card reader receives the identity number. If the variable blink interval is adopted, the maximum transmission interval between two signals could be 2 times the average interval. Then:

$2 n_{t} \times \overline{T_{B I}}<\frac{2 d}{V}$, i.e., $\overline{T_{B I}}<\frac{d}{v \times n_{t}}$

Through the above analysis, it is known that the value range of the average interval $\overline{T_{B I}}$ should be $\left(\frac{T \times E_{t}}{E}, \frac{d}{v \times n_{t}}\right)$. To get the proper average interval, the middle and lower limit of the value range should be smaller than the upper limit, i.e.:

$$
\frac{T \times E_{t}}{E}<\frac{d}{v \times n_{t}}
$$

According to Formula (6), the electric quantity of the battery provided for the tag should be $E>\frac{v n_{t} T E_{t}}{d}$.

It has been known that the total time interval during which there is no consecutive collision allowed within the average time interval should be $\left[\frac{T_{B I}}{2 t s}\right]$.

Let $q \times N \leq\left[\frac{T_{B I}}{2 t s}\right]$

In Formula (7), $N$ is used for complementation of the identity number. $q$ will be used for adjustment of the maximum number of tags allowed in the identification area. The calculation method of the connection interval $T_{B I}$ of the tag for the $r$ time should be as follows:

$T_{B I}(r)=\left\{\begin{array}{l}\overline{T_{B I}}+\left(I D \% N+r^{\prime} \times N\right) \times 2 t s \quad r^{\prime}<q \\ \overline{T_{B I}}-\left[I D \% N+\left(r^{\prime}-q\right) \times N\right] \times 2 t s \quad r^{\prime} \geq q\end{array}\right.$

In formula (8), $r^{\prime}=r \% 2 q . I D$ is the identity number of the tag. The algorithm allows each tag to value alternately within $2 q$ different blink intervals.

\subsection{Analysis Of Algorithm Performance}

Let the remainder of ID of any two tags (tags A and B) within the identification area of a card reader minus $N$ be $i d_{a}$ and $i d_{b}$ respectively and $i d_{a} \neq i d_{b}$, and then the difference between the possible value of the blink interval of tags A and B calculated according to the algorithm is

$\left|T_{B I a}-T_{B I b}\right|=\left|i d_{a} \pm i d_{b}\right| \times 2 t_{s}+h N \times 2 t_{s}$
In Formula (9), $h$ is a random nonnegative integer, and is related to the number of times of transmission by tags $\mathrm{A}$ and $\mathrm{B}$ and the value of $q$. Within the possible value range of $h$, as $i d_{a} \neq i d_{b}$, then $\left|T_{B I a}-T_{B I b}\right| \geq 2 t_{s}$. It can be known that if there is collision between any of the signals transmitted by two tags, then there will not be collision next time. If $I D$ equals after complementation, i.e.. $i d_{a}=i d_{b}$, when the tags are in the same identification area and there is collision, then there will only be collision when the interval of the next blink are the same, which means the conditional probability of consecutive collision is $\frac{1}{2 q}$. To avoid this situation, consecutive numbers could be used for coding when setting the identity number, and the value range of $N$ could be expanded.

Through the above analysis, it can be known that the algorithm meets the following requirements: (1) When more than one tag is within the receiving range of one card reader, the consecutive collision between any two signals could be effectively avoided. If the two tags collide with each other during a signal transmission, there will not be collision between the two tags in the next transmission. (2) The same average blink interval could be preset for all tags. This allows the energy consumption of tags in the same batch to be basically the same. They can be changed in a batch when the working hour upper limit is reached.

\section{SIMULATION AND RESULT ANALYSIS}

\subsection{Simulation Environment}

This paper realized the blink interval generation algorithm using the simulation tool MATLAB, and compared the algorithm with the ALOHA protocol and INCITS371.1 protocol. To fully reflect the omission difference caused by blink intervals, the blink times within a unit time for each interval generation algorithm is the same. Specifically, the ALOHA protocol tag transmits the signal once during the average time interval; INCITS371.1 protocol allows transmission of 1-4 times during each interval. To facilitate comparison, the number of transmissions during one interval is one, and the computing method for the interval is by summing up the random values distributed evenly between $-638 \mathrm{~ms}-638 \mathrm{~ms}$ and the average time interval. In the simulation, assume all tags are put together, and the communication transmission bit rate between the tag and the card reader is $250 \mathrm{kbps}$. The frame size of identity number carried by each transmitted signal is $80 \mathrm{bit}$. The frequency offset of the tag clock is 40ppm, See in Fig. (2).

\subsection{Simulation Result Analysis}

According to the AQ 6210-2007 General Technical Condition for Management System of Workers Working in the Underground Coal Mines, the omission rate of the realtime location system should be no more than $10^{-4}$. Assume the number of tags passing through the identification range of the same card reader is 80 , and then the average blink interval of the tag is $2 \mathrm{~s}$. In the experiment, the length of card 
reading cycle of the card reader was gradually increased, until the requirement that the omission rate should be less than $10^{-4}$ is met.

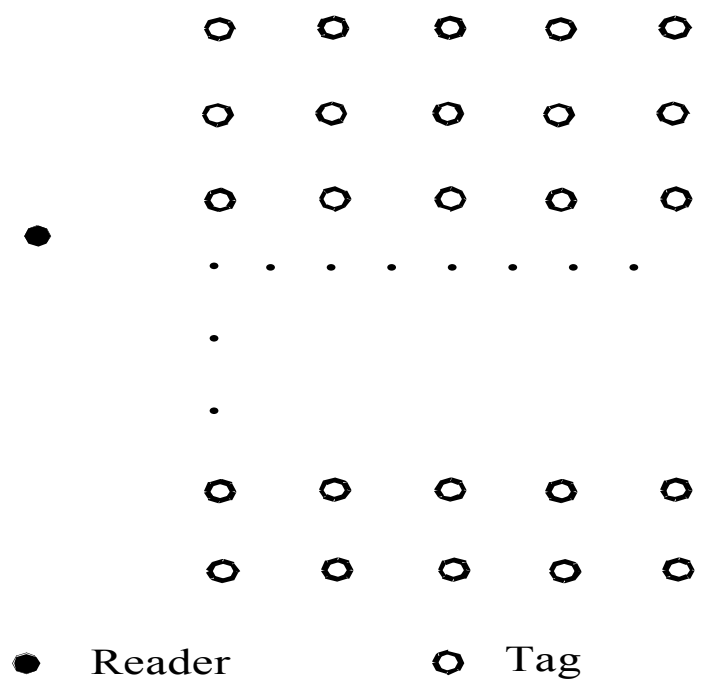

Fig. (2). Simulation environment.

Fig. (3) shows the omission rate changed with different blink interval. When the value of parameter $q$ of algorithm IBBIGA is 2 or 3 , the omission rate is also the same, and is lower than that of ALOHA and INCITS371.1 in which the random method is used to generate blink intervals. In the simulation, the value of $q$ is 0 . This indicates that the blink interval of each tag is the same, being $2 \mathrm{~s}$. When the value of $q$ is 0 or 1 , the selected range of the blink interval of the tag is too small, which will result in a high possibility of consecutive collision. In such a case, the omission rate can not reach the requirement.

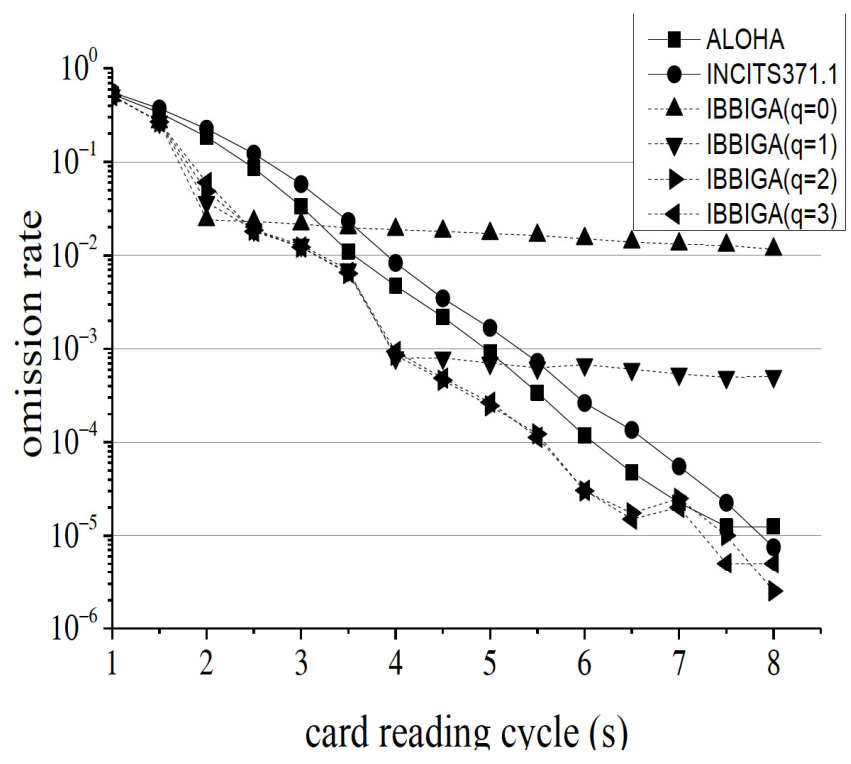

Fig. (3). Omission rate versus blink interval.
Fig. (4) shows the blink times of tags in the three different protocols during the simulation. To facilitate comparison, the data has been normalized. According the requirement of the simulation, the average blink times of tags are equal. As the ALOHA protocol and INCITS 371.1 protocol randomly calculate next blink time, after a long period the blink times between different tags will become diversity. This will cause disequilibrium of energy consumption to the different tags. The IBBIGA protocol calculate blink time by identity number, this can keep each tag has the same blink times no matter how long time is elapsed.

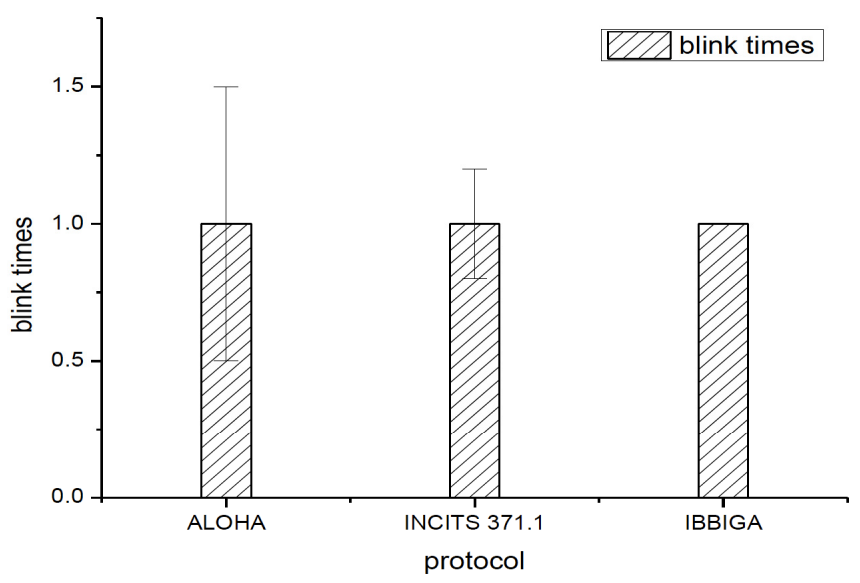

Fig. (4). Blink times comparison of different protocol.

Table 1 shows the relation between the blink interval and the card reading cycle along with change of the number of tags when the omission rate is 10-4. It can be seen that as the average interval increases, the IBBIGA protocol performance gradually improves. When the number of tags within the identification area is between $80 \sim 200$, the card reading cycle is $7 \%$ lower than ALOHA and 12\% lower than INCITS371.1 averagely in the tag location system in which the blink interval is generated based on the identity number.

\section{CONCLUSION}

To reduce the tag reading omission caused by consecutive collision of signals in the real-time location system used in underground coal mines, this paper proposed a blink interval computing method based on identity number. In this algorithm, no random number needs to be generated. The uniqueness of the identity number is used to generate a set of corresponding blink intervals. This algorithm could ensure that if the signals transmitted by any two tags collide with each other, then the signals transmitted by these two tags the next time will not collide with each other. In addition, the average connection interval of each tag will be the same. Through simulation analysis, when the average number of times of transmission in the unit time is the same, the algorithm will have a lower omission rate. When the number of tags in the identification scope is $80 \sim 200$, the he 
Table 1. Average blink interval versus term of reading.

\begin{tabular}{|c|c|c|c|c|c|}
\hline Node number & Algorithm & $\overline{T_{B I}}=1 \mathrm{~s}$ & $\overline{T_{B I}}=2 \mathrm{~s}$ & $\overline{T_{B I}}=3 \mathrm{~s}$ & $\overline{T_{B I}}=4 \mathrm{~s}$ \\
\hline & INCITS371.1 & 3.9 & 6.5 & 8.9 & 11.5 \\
\hline \multirow[t]{3}{*}{80} & ALOHA & 3.6 & 6.1 & 8.5 & 10.9 \\
\hline & $\operatorname{IBBIGA}(q=2)$ & 3.4 & 5.6 & 7.5 & 9.5 \\
\hline & INCITS371.1 & 4.2 & 6.9 & 9.9 & 12.2 \\
\hline \multirow[t]{3}{*}{120} & ALOHA & 3.9 & 6.6 & 9.2 & 11.5 \\
\hline & $\operatorname{IBBIGA}(q=2)$ & 3.9 & 6.1 & 8.5 & 11.0 \\
\hline & INCITS371.1 & 4.7 & 7.5 & 10.3 & 12.6 \\
\hline \multirow[t]{3}{*}{160} & ALOHA & 4.4 & 7.1 & 9.7 & 11.9 \\
\hline & $\operatorname{IBBIGA}(q=2)$ & 4.1 & 6.3 & 8.9 & 11.3 \\
\hline & INCITS371.1 & 5.0 & 8.0 & 10.8 & 13.3 \\
\hline \multirow[t]{2}{*}{200} & ALOHA & 4.7 & 7.6 & 10.3 & 12.6 \\
\hline & $\operatorname{IBBIGA}(q=3)$ & 4.6 & 7.3 & 9.3 & 11.8 \\
\hline
\end{tabular}

card reading cycle with an omission rate of $10-4$ is $7 \%$ lower than the pure ALOHA and 12\% lower than INCITS 371.

\section{CONFLICT OF INTEREST}

The author(s) confirm that this article content has no conflict of interest.

\section{ACKNOWLEDGEMENTS}

This research project is a project under the National Scitech Support Plan (2012BAH12B01). A major natural science research project of colleges and universities in Jiangsu (13KJA520007). This project is supported by the Innovation Plan of Postgraduates in Ordinary Colleges and Universities of Jiangsu Province (CXZZ11_0291).

\section{REFERENCES}

[1] P.K. Mishra, et al., "RFID in underground-Mining Service applications," IEEE pervasive computing. vol. 13, pp. 72-79, 2014

[2] C.C. Ye, et al., "Personnel Positioning System of Underground Mines Based on RFID," Journal of Wuhan University of Technology. vol. 32, pp. 146-149, 2015
[3] N. Björn, et al., "An energy and application scenario aware active RFID protocol," EURASIP Journal on Wireless Communications and Networking, vol. 2010,2010

[4] A. Agarwal, et al., "Real time location estimation using active RFID system," 2008 International Conference of Recent Advances in Microwave Theory and Applications. pp. 540-542, Nov. 2008

[5] B. Tas, et al., "Data Collection Using Transmit-Only Sensors and a Mobile Robot in Wireless Sensor Networks," International Conference on Computer Communications and Networks. pp. 1-9, Aug. 2012

[6] Z. Jia, et al., "Improve Efficiency and Reliability in Single-Hop WSNs with Transmit-Only Nodes,". IEEE TRANSACTIONS ON PARALLEL AND DISTRIBUTED SYSTEMS. vol. 24, pp. 520-534, 2013

[7] E. Egea-Lopez, et al., "Performance evaluation of non-persistent CSMA as anti-collision protocol for active RFID tags," 5th International Conference on Wired/Wireles Internet Communications. Coimbra, Portugal. pp. 279-289. 2007.

[8] Y. Zhang, "A new dynamic frame slotted ALOHA-algorithm for anti-collision in RFID systems," China-Japan Joint Microwave Conference. pp. 502-506, 2008

[9] R.S. Sudhaakar, et al., "A novel QoS-aware MAC scheme using optimal retransmission for wireless networks,". IEEE Transactions on Wireless Communications. vol. 8, pp.2230-2235, 2009

[10] S. Feng, et al., "Review of Studies of Tag Anti-collision Algorithm in RFID," 2009 International Conference on Wireless Networks and Information Systems. pp. 121-125, 2009

[11] Y. Ji, et al., "Concurrent collision probability of rfid tags in underground mine personnel position systems," Mining Science and Technology, vol. 20, pp. 734-737, 2010 